\title{
Secure Signature Schemes based on Interactive Protocols
}

\author{
Ronald Cramer (CWI, Amsterdam, cramer@cwi.nl), \\ Ivan Damgård (BRICS *, Aarhus University, ivan@daimi.aau.dk)
}

\begin{abstract}
Given only an interactive protocol of a certain type as a primitive, we can build a (non-interactive) signature scheme that is secure in the strongest sense of Goldwasser, Micali and Rivest (see [11]): not existentially forgeable under adaptively chosen message attacks. There are numerous examples of primitives that satisfy our conditions, e.g. Feige-Fiat-Shamir, Schnorr, Guillou-Quisquater, Okamoto and BrickellMc.Curley ([9], [17], [12], [15], [3]).

A main consequence is that efficient and secure signature schemes can now also be based on computationally difficult problems other than factoring (see [11]), such as the discrete logarithm problem.

In fact, the existence of one-way group homomorphisms is a sufficient assumption to support our construction. As we also demonstrate that our construction can be based on claw-free pairs of trapdoor permutations, our results can be viewed as a generalization of [11].
\end{abstract}

\section{Introduction}

This paper deals with the construction of secure signature schemes. By "secure", we mean that some well-defined computational assumption can be shown to be sufficient for the scheme not to be existentially forgeable, even under an adaptive chosen message attack. This notion of optimal security was introduced in [11]. Most, if not all, signature schemes used in practice such as ISO9796/RSA or DSA are based on a computational assumption that is certainly necessary for this kind of security, but not known to be sufficient.

Goldwasser, Micali and Rivest [11] were the first to find a provably secure signature scheme, based on the existence of claw-free pairs of trapdoor permutations. Merkle [13] showed essentially that existence of collision intractable hash functions is a sufficient assumption. Naor and Yung showed that any one-way permutation is also enough [14], and finally this was reduced to any one-way function (which is also a necessary assumption) by Rompel [16].

Although secure signature schemes are generally less efficient than the ones used in practice, the efficiency of the GMR scheme is not too bad when based on factoring. Measured in signature length and time for signature generation, GMR is worse than plain RSA by a factor of 5-20, depending on the number of messages to be signed. By relying on the (perhaps) stronger assumption that

\footnotetext{
* Basic Research in Computer Science, Centre of the Danish National Research Foundation
} 
RSA is hard to invert, Bos and Chaum [2] have been able to build an even more efficient secure scheme. Dwork and Naor [7] have exhibited an efficient and secure signature scheme whose security is also equivalent to the difficulty of RSAinversion. In contrast with other schemes that use authentication trees, such as [11], they are able to re-use the authenticating nodes many times. As a result of this and further exploitations of the specific properties of the RSA functions, the length of their signatures can be made quite small, although a price has to be paid in the form of a large public file. In [6], similar trade-offs between shared random strings and the size of secure signatures have been achieved for certain families of claw-free pairs of trapdoor permutations, in particular a family based on the dificulty of factoring integers.

On the theoretical side, the reduction in the necessary assumptions by [13], [14] and [16] have come at the price of dramatically reduced efficiency. In particular, signatures have become larger. Where a GMR signature is of length $O(k \log i)$ bits, where $k$ is the security parameter and $i$ indicates the number of signatures made, a Naor-Yung signature would typically be of length $O\left(k^{2} \log i\right)$ bits, because a full preimage under a one-way function is required to authenticate 1 bit.

Thus it has been an open question whether secure signatures with efficiency comparable to or better than that of GMR could be based on more general assumptions than claw-free pairs of trapdoor permutations.

In this paper, we show that secure signature schemes with signatures as short as those of GMR can be built if so called signature protocols exist. In particular, our schemes have the same property as GMR that the length of signatures grow logarithmically with the number of messages signed. Note, however, that Goldreich [10] has shown that the GMR scheme can be modified so that all signatures have length $O(k \log k)$ bits. This same modification applies to our scheme as well. Note also that Goldreich's modification makes the signature scheme memoryless, which implies that a signature will not reveal the number of signatures made so far.

Dropping some technical details, a signature protocol is an interactive protocol for a hard problem that uses three messages, where the prover speaks first and the verifier sends a random challenge as the second message. The essential properties are

-- The protocol must be secure (zero-knowledge) against the honest verifier.

- The challenge must be longer than the prover's first message.

- It must be infeasible for a cheating prover to answer more than one challenge in a given protocol execution.

Furthermore, we show that it is sufficient for the existence of signature protocols (and hence for the existence of secure signatures) that one-way group homomorphisms exist. This has a nice theoretical consequence, because it shows that, compared to GMR, the trapdoor property can be traded for the homomorphism property without getting longer signatures. Moreover, our construction allows us, in both signature generation and verification, to minimize the number 
of evaluations of the one-way function and replace them by evaluations of the group operation in the groups involved. This means that we can use the discrete logarithm assumption as a basis for secure signatures in a much more efficient way than known before. Where earlier methods would, with security parameter $k$, require $O\left(k^{2}\right)$ exponentiations per basic authentication step and give signatures of length $O\left(k^{2} \log i\right)$ bits, our method requires $O(1)$ exponentiations and gives signatures of length $O(k \log i)$.

We also show that existence of a three pass public coin proof of knowledge for any hard problem ( $A$ hard random self-reducible problem would be enough for this) and a collision intractable hash function implies existence of signature protocols. Although the hash function alone would be enough to construct secure signatures, using our method may lead to shorter signatures $(O(k \log i)$ compared to $\left.O\left(k^{2} \log i\right)\right)$, depending on the protocol used.

\section{Signature Protocols}

$P$

$(a, \operatorname{aux}(a)) \leftarrow P_{a}(x, w)$

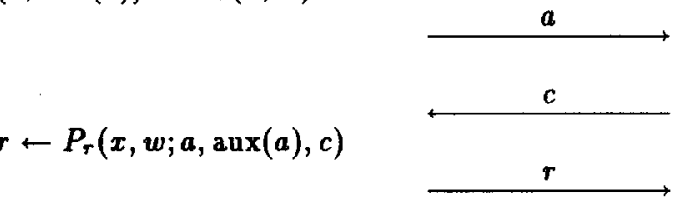

$V$

$$
c \leftarrow\{0,1\}^{C_{P}}
$$

$\phi(x, a, c, r) \stackrel{?}{=} 1$

Fig. 1. Protocol $\mathcal{P}$, common input $x$, private input for $P$ is $w$

This section is devoted to defining the basic building block, a signature protocol, that is used in our construction for secure signatures. Let $\mathcal{P}$ be a three round public coin protocol where the prover speaks first. Figure 1 depicts the kind of protocol we will look at. It resembles a proof of knowlege for a binary relation $R$ (see for instance [8] for details), in that the prover can always make the verifier accept on common input $x$, if the prover knows $w$ such that $(x, w) \in R$.

Indeed, by running (probabilistic) polynomial time algorithm $P_{a}$ on $x$ and his secret witness $w$, the prover $P$ computes his initial message $a$, and some (secret) auxiliary information aux $(a)$. The length of this first message $a$ is denoted $A_{\mathcal{P}}$, the authentication length, which only depends on $x$. After having received $a$, the verifier $V$ chooses a challenge $c$ uniformly at random, and sends it to $P$. The length of admissible challenges in $\mathcal{P}$ is called the challenge length $C_{\mathcal{P}}$ (we will sometimes abuse this notation to refer to the set of possible challenges). Also here, it is assumed to depend only on $x$. The prover $P$ completes the conversation 
by running (probabilistic) polynomial time algorithm $P_{r}$ on $x, w, a, c$, and, the auxiliary information $\operatorname{aux}(a)$ for $a$. The resulting response $r$ is submitted to the verifier $V$. We will assume that the procedure $\phi$ that the verifier $V$ invokes to test the validity of the conversation, is a polynomial time algorithm. The collection of all possible accepting conversations with respect to $x$ will be denoted $A c c(x)$. For the rest of this paper, $\mathcal{P}$ will denote a protocol as described above.

For the purpose of constructing secure signature schemes, we require the following from $\mathcal{P}$ in stead of the ordinary soundness condition:

Definition 1 Let $k$ be a security parameter for protocol $\mathcal{P}$. Suppose we are given a probabilistic polynomial time generator $G$ for relation $R$ that on input $1^{k}$ produces $(x, w) \in R$, such that no probabilistic polynomial time algorithm, given $x$ as input, can generate two accepting conversations (with respect to $x)(a, c, r)$, $\left(a, c^{\prime}, r^{\prime}\right)$ from $\operatorname{Acc}(x)$, with $c \neq c^{\prime}$, except with negligible probability of success. Then $\mathcal{P}$ is called collision intractible over $G$.

Next, we need the protocol $\mathcal{P}$ to be honest verifier zero-knowledge, that is, we only demand that conversations with a verifier who follows protocol $\mathcal{P}$ can be simulated. Additionally, we require that the simulator outputs accepting conversations where the challenge can be chosen in advance, i.e., the simulator can take any value $c$ as input, and will output an accepting conversation where the challenge is equal to $c$. A protocol $\mathcal{P}$ satisfying these conditions will be called special honest verifier zero-knowledge.

More precisely, let $(x, w) \in R$ and let a prover $P$ and a verifier $V$ with common input $x$ be given. The prover has $w$ as private input. Then $\mathcal{P}(x, w)$ denotes the probability distribution on $A c c(x)$ induced by conversations between $P$ and $V$, provided that they both follow protocol $\mathcal{P}$ honestly. We require the following:

Definition 2 Let $(x, w) \in R$. Suppose we are given a probabilistic polynomial time algorithm $\mathcal{S}$ with the following properties.

1. On input $x$ and any $c \in C_{\mathcal{P}}, \mathcal{S}$ outputs an accepting conversation from $\operatorname{Acc}(x)$, where $c$ is the challenge.

2. The distribution of $\mathcal{S}(x, c)$, where $c$ is chosen uniformly at random from $C_{\mathcal{P}}$, is equal to $\mathcal{P}(x, w)$.

Then $\mathcal{P}$ is called special honest verifier zero knowledge, and $\mathcal{S}$ its special simulator.

In the following we will demonstrate that a protocol $\mathcal{P}$ that is special honest verifier zero-knowledge, is in fact secure against a slightly more general verifier. It follows immediately from Definition 2 that, for each fixed $c \in C_{\mathcal{P}}, \mathcal{S}(x, c)$ outputs conversations $(a, c, r) \in A c c(x)$ with exactly the same distribution as $(a \leftarrow$ $\left.P_{a}(x, w), c, r \leftarrow P_{r}(x, w, a, \operatorname{aux}(a), c)\right)$, i.e., according to the honest prover who has access to $(x, w)$. Therefore, in order for the conversations to be simulatible, it is sufficient that the verifier chooses challenges $c$ independently from the first message in any given execution of $\mathcal{P}$. This proves the following theorem: 
Theorem 1 If $\widetilde{V}$ is any probabilistic polynomial time verifier who, in any given execution of protocol $\mathcal{P}$, chooses the challenge $c$ independently from the prover's first message a, then the conversation between prover $P$ and verifier $\tilde{V}$ can be simulated by means of the special simulator $\mathcal{S}$.

Summarizing, we require the following of our protocol $\mathcal{P}$ in order for it to support our construction of (non-interactive) secure signature schemes.

Definition 3 Suppose $\mathcal{P}$ satisfies the following conditions.

1. $C_{\mathcal{P}}>A_{\mathcal{P}}$.

2. $\mathcal{P}$ is collision-intractible over $G$.

3. $\mathcal{P}$ is special honest verifier zero-knowledge.

Then $\mathcal{P}$ is called a signature protocol. If $\mathcal{P}$ satisfies the second condition and is honest verifier zero-knowledge (so it does not necessarily have a special simulator), $\mathcal{P}$ is called a quasi signature protocol.

The following theorem shows that any given signature protocol $\mathcal{P}$ can be transformed into a new signature protocol $\mathcal{P}^{*}$ where the challenge length $C_{\mathcal{P}}$ * can be of any size polynomial in the security parameter $k$.

Theorem 2 Suppose there exists a signature protocol $\mathcal{P}$ for relation $R$ and generator $G$, then there is a signature protocol $\mathcal{P}^{*}$ for $R$ and $G$, satisfying that $C_{\mathcal{P}^{*}}=t$, for any $t$ polynomial in the security parameter $k$.

Proof. Without loss of generality, we may assume that $A_{\mathcal{P}}+1=C_{\mathcal{P}}$ The protocol $\mathcal{P}^{*}$ goes as follows:

1. The prover sends a first message $a$ to the verifier, where $a$ is computed as in $\mathcal{P}$.

2. The verifier sends $t$ random bits $b_{1}, \ldots, b_{t}$.

3. The prover sends $t$ conversations in $\mathcal{P},\left(a_{i}, c_{i}, r_{i}\right), i=1, \ldots, t$, where $c_{i}=$ $b_{i} \| a_{i+1}$ for $i=1, \ldots, t-1$ and $c_{t}=b_{t}\|0\| \cdots \| 0$.

4. The verifier checks that $a=a_{1}$, that all conversations are accepting conversations, and that $c_{i}=b_{i} \| a_{i+1}$ for $i=1, \ldots, t-1$, and that $c_{t}=b_{t}\|0\| \cdots \| 0$.

By construction, the challenge length $t$ for $\mathcal{P}^{*}$ can be chosen what we want it to be, provided $t=\operatorname{poly}(k)$. Suppose now that we are given two accepting conversations in $\mathcal{P}^{*}$ for some public string $x$ with the same first message $a$, but with different challenges $\left(b_{1}, \ldots, b_{t}\right)$ and $\left(b_{1}^{\prime}, \ldots, b_{t}^{\prime}\right)$. Let, for $j=1 \ldots t$, $\left(a_{j}, c_{j}, r_{j}\right)$ and $\left(a_{j}^{\prime}, c_{j}^{\prime}, r_{j}^{\prime}\right)$ be the respective replies in those conversations in $\mathcal{P}^{*}$, and let $i$ be an index such that $b_{i} \neq b_{i}^{\prime}$. Clearly, this implies that $c_{i} \neq c_{i}^{\prime}$. Take $i$ to be the smallest index such that $c_{i} \neq c_{i}^{\prime}$. If $i=1$, we have a collision in $\mathcal{P}$ with respect to $x$, as by definition of $\mathcal{P}^{*}$, we must have $a_{1}=a_{1}^{\prime}=a$.On the other hand, if $i>1, c_{i-1}$ must be equal to $c_{i-1}^{\prime}$, i.e., $b_{i-1}\left\|a_{i}=b_{i-1}^{\prime}\right\| a_{i}^{\prime}$. But then $a_{i}=a_{i}^{\prime}$ and we have a collision $\left(a_{i}, c_{i}, r_{i}\right),\left(a_{i}^{\prime}, c_{i}^{\prime}, r_{i}^{\prime}\right)$ in $\mathcal{P}$ with respect to $x$. Therefore, $\mathcal{P}^{*}$ is collision-intractible over $R$ and $G$. 
As for special honest verifier zero-knowledge of $\mathcal{P}^{*}$, we now exhibit a special simulator $\mathcal{S}^{*}$ for $\mathcal{P}^{*}$, that runs $\mathcal{S}$ as a subroutine. $\mathcal{S}^{*}$ starts by receiving a public string $x$ and a challenge $\left(b_{1}, \ldots, b_{t}\right)$ as input. It proceeds by putting $c_{t}=b_{t}\|0\| \cdots \| 0$, and feeding $x$ and $c_{t}$ to $\mathcal{S}$. After $\mathcal{S}$ has output an accepting conversation $\left(a_{t}, c_{t}, r_{t}\right)$ in $\mathcal{P}$ with respect to $x, \mathcal{S}^{*}$ repeats the following for $i=t-1 \ldots 1$. Put $c_{i}=b_{i} \| a_{i+1}$, feed $x$ and $c_{i}$ to $\mathcal{S}$ and receive an accepting conversation $\left(a_{i}, c_{i}, r_{i}\right)$ from $\mathcal{S}$. By invoking Theorem 1 , it is clear that $\mathcal{S}^{*}$ generates accepting conversations in $\mathcal{P}^{*}$ with respect to $x$, with exactly the same distribution as the conversations with the honest verifier in $\mathcal{P}^{*}$.

Thus, in the constructions to follow, whenever we have a signature protocol, we may assume that the challenge length is whatever we need it to be. Before investigating under which general assumptions signature protocols can be shown to exist, we mention Guillou-Quisquater [12], Okamoto [15] (both the factoring and the RSA-versions) and Fiat-Shamir [9] (if the number of secret roots is chosen sufficiently large) as examples of proofs of knowledge that can be viewed as signature protocols. Schnorr's discrete log protocol [17] does not directly satisfy the conditions, but can be modified to do so since it is based on a one-way group homomorphism (see below).

\section{Sufficient Assumptions}

The most general computational assumptions we have been able to find, sufficient for existence of signature protocols, is the existence of one-way group homomorphisms, and the existence of claw-free pairs of trapdoor permutations. No implication is known in either direction between these two assumptions.

\section{One-Way Group Homomorphisms}

Definition 4 A family of one-way group homomorphisms is a family of group homomorphisms $\mathcal{F}=\{f: G \rightarrow H\}$. In the following, we let $k_{f}=\log _{2}(|H|)$, i.e. the number of bits needed to represent an element in $H$. We will sometimes drop subscript $f$, if it is clear which $f$ we refer to. The family has to satisfy the following properties:

1. There is a polynomial time algorithm which given $f$ and $w \in G$, computes $f(w)$ in time polynomial in $k$.

2. There is a probabilistic polynomial time algorithm which on input $1^{k}$ outputs an element $f: G \rightarrow H$ chosen uniformly from $\mathcal{F}$, subject to $k=k_{f}$.

9. The elements $f: G \rightarrow H \in \mathcal{F}$ satisfy that there is a probabilistic algorithm which given $G$ outputs an element chosen uniformly from $G$, in time polynomial in $k$.

4. The one-way property: Let $A$ be any prababilistic polynomial time algorithm which receives input $f$ and $f(w)$, where $f, w$ are chosen as in points 2 and 3. Then the probability that $A$ outputs $y$ such that $f(y)=f(w)$ is superpolynomially small in $k$. 
5. The elements $f: G \rightarrow H \in \mathcal{F}$ satisfy that group operation and inversion in $G$ and $H$ can be computed in time polynomial in $k$.

Examples of possible one-way group homomorphisms are the RSA functions, squaring modulo a composite number, or discrete exponentiation modulo a prime, or on an elliptic curve. Given a family as in this definition, we can make the following binary relation and generator for it:

Definition 5 Let $\mathcal{F}$ be as in Definition 4. Then $R_{\mathcal{F}}$ is the binary relation consisting of pairs $\left(\left(f, x_{1}, \ldots, x_{k_{f}+1}\right),\left(w_{1}, \ldots, w_{k_{f}+1}\right)\right)$, where $f \in \mathcal{F}$ and $f\left(w_{i}\right)=$ $x_{i} . G_{F}$ is the generator that on input $1^{k}$ generates $f$ using property 2 of Definition 4, generates $w_{1}, \ldots, w_{k_{f}+1}$ using property 9 and finally computes $x_{i}=f\left(w_{i}\right)$.

Theorem 3 Suppose $\mathcal{F}$ is a family of one-way group homomorphisms. Then there exists a signature protocol for $R_{\mathcal{F}}$ and $G_{\mathcal{F}}$.

Proof. The protocol claimed takes $f, x_{1}, \ldots, x_{k+1}$ as common input, while the witnesses $w_{1}, \ldots, w_{k+1}$ are private input to the prover. The protocol is now a straightforward generalization of Feige-Fiat-Shamir [9] and goes as follows:

1. The prover chooses a random $r \in G$ and sends $f(r)$ to the verifier.

2. The verifier chooses bits $e_{1}, \ldots, e_{k+1}$ at random and sends them to the prover.

3. The prover returns $z=r \cdot w_{1}^{e_{1}} \cdots w_{k+1}^{e_{k+1}}$. The verifier checks that $f(z)=$ $f(r) \cdot x_{1}^{e_{1}} \cdots x_{k+1}^{e_{k+1}}$

This protocol is clearly complete with probability 1 . Honest verifier zero knowledge is clear by standard arguments: first choose $z$ and $e_{1}, \ldots, e_{k+1}$ at random, then use this to compute an $f(r)$-value. It is also clear that the challenge is one bit longer than the first message from the prover. Thus, only the collision intractable property remains to be argued. Assume by contradiction that some enemy $A$ can produce $z, z^{\prime}$ and $\left(e_{1}, \ldots, e_{k+1}\right) \neq\left(e_{1}^{\prime}, \ldots, e_{k+1}^{\prime}\right)$ such that $f(z)=f(r) \cdot x_{1}^{e_{1}} \cdots x_{k+1}^{e_{k+1}}$ and $f\left(z^{\prime}\right)=f(r) \cdot x_{1}^{e_{1}^{\prime}} \cdots x_{k+1}^{e_{k+1}^{\prime}}$. This means that

$$
f\left(z \cdot z^{\prime-1}\right)=x_{1}^{d_{1}} \cdots x_{k+1}^{d_{k+1}},
$$

where all $d_{i}$ are $1,-1$ or 0 , and at least one of them is non-zero.

We can then build the following algorithm which will invert $f$ with the help of $A$ : given a random $f$-image $x$, generate an output seemingly coming from $G_{\mathcal{F}}$ as follows: choose $w_{1}, \ldots, w_{k+1}$ and $1 \leq j \leq k+1$ at random. Put $x_{i}=f\left(w_{i}\right)$ for $i \neq j$, and $x_{j}=f\left(w_{j}\right) \cdot x$. Now run $A$ 's algorithm with $f$ and the $x_{i}$ 's as input. Clearly the set of $x_{i}$ is distributed exactly as output from $G_{\mathcal{F}}$, whence $A$ 's success probability is the same as in real life. Note that if $A$ has success, we can write $x^{d_{j}}$ as

$$
x^{d_{j}}=f\left(z \cdot z^{\prime-1} \cdot \prod_{i} w_{i}^{-d_{i}}\right)
$$

Now note that the set of $x_{i}$ 's contains no information about $j$, whence the probability that $d_{j} \neq 0$, given that $A$ has success, is at least equal to $1 /(k+1)$. 
Remark 1 It is clear that the protocol constructed in the proof above can be modified to have any challenge length desired by having more $x_{i}$-values. Enlarging the challenge length in this way will be more efficient than using Theorem 2.

We briefly state that, in the definition of a signature protocol, we can exchange the assumption on the challenge length and the existence of a special simulator for the assumption that we are given a family of collision-intractable hash-functions, as can be seen from the following theorems. The proofs are given in [5].

Theorem 4 Let $\mathcal{P}$ be honest verifier zero-knowledge and collision-intractable over $R$ and $G$. Then $\mathcal{P}$ can be compiled into a protocol $\mathcal{P}^{*}$ (for relation $R$ and generator $G$ ), that is also collision-intractable over $R$ and $G$ but that additionally satisfies special honest verifier zero-knowledge.

Theorem 5 Suppose there exists a quasi signature protocol $\mathcal{P}$ for relation $R$ and generator $G$ and that a family $\mathcal{H}$ of collision intractable hash functions exists. Then there exists a signature protocol $\mathcal{P}^{*}$ for $R_{\mathcal{H}}$ and $G_{\mathcal{K}}$. Here $R_{\mathcal{H}}$ consists of pairs $((x, h), w)$ where $(x, w) \in R, w$ is of length $k$ bits and $h \in \mathcal{H}$ has output length $k$. The generator $G_{\mathcal{H}}$ runs $G$ to generate $(x, w)$ and then selects $h \in \mathcal{H}$ with the desired output length.

\section{Claw-Free Pairs of Trapdoor Permutations}

In [11], a secure signature scheme is exhibited, based on (a family of) claw-free pairs of trapdoor permutations. The following theorem is proved in [5].

Theorem 6 Suppose that a family of claw-free pairs of trapdoor permutations exists. Then there exists a signature protocol.

\section{Main Result}

We will now present the new signature scheme $\Sigma_{\mathcal{p}}$, based on a signature protocol $\mathcal{P}$. In Section 5 , we prove that $\Sigma_{\mathcal{P}}$ is not existentially forgeable under adaptively chosen message attacks. A concrete example is given in Section 6.

Theorem 7 Let $\mathcal{P}$ be a signature protocol for relation $R$ and generator $G$. Then the signature scheme $\Sigma_{\mathcal{P}}$ is not existentially forgeable under adaptively chosen message attacks.

It is assumed that we are given a signature protocol $\mathcal{P}$ for relation $R$ and generator $G$. By Theorem 2, we may assume that for each security parameter $k$ and for each instance $(x, w)$ as output by running $G\left(1^{k}\right)$, the (non-constant) polynomial $t(k)$ satisfies $t=C_{\mathcal{P}} \geq 3 \cdot A_{\mathcal{P}}$. The construction of $\Sigma_{\mathcal{P}}$ from $\mathcal{P}$ works as follows. 


\section{Initialization Phase}

Given a security parameter $k$, the signer uses the generator $G$ to generate two solved instances $x_{0}$ and $x_{1}$, with respective witnesses $w_{0}$ and $w_{1}$. He also computes $\left(a_{1}^{1}, \operatorname{aux}\left(a_{1}^{1}\right)\right) \leftarrow P_{a}\left(x_{1}, w_{1}\right)$ and puts $\left(x_{0}, x_{1}, a_{1}^{1}\right)$ in his public directory.

\section{Signing Phase}

Let $m \in\{0,1\}^{t}$ be the message to be signed and let $i \geq 1$. The $i$-th signature, on a message $m \in\{0,1\}^{t}$, is computed as follows. First, the signer computes

1. $\left(a_{0}^{i}, \operatorname{aux}\left(a_{0}^{i}\right)\right) \leftarrow P_{a}\left(x_{0}, w_{0}\right)$,

2. $r_{0}^{i} \leftarrow P_{r}\left(x_{0}, w_{0} ; a_{0}^{i}, \operatorname{aux}\left(a_{0}^{i}\right), m\right)$,

3. $\left(a_{1}^{2 i}, \operatorname{aux}\left(a_{1}^{2 i}\right)\right) \leftarrow P_{a}\left(x_{1}, w_{1}\right),\left(a_{1}^{2 i+1}, \operatorname{aux}\left(a_{1}^{2 i+1}\right)\right) \leftarrow P_{a}\left(x_{1}, w_{1}\right)$,

4. $r_{1}^{i} \leftarrow P_{r}\left(x_{1}, w_{1} ; a_{1}^{i}, \operatorname{aux}\left(a_{1}^{i}\right), a_{1}^{2 i}\left\|a_{1}^{2 i+1}\right\| a_{0}^{i}\right)$.

The signer stores $a_{1}^{2 i}, \operatorname{aux}\left(a_{1}^{2 i}\right), a_{1}^{2 i+1}, \operatorname{aux}\left(a_{1}^{2 i+1}\right), a_{0}^{i}, r_{1}^{i}$, Let Auth $\left(a_{0}^{i}\right)$ be an authentication path for $a_{0}^{i}$, i.e., Auth $\left(a_{0}^{i}\right)$ consists of all tuples of the form $\left(a_{1}^{j}, a_{1}^{2 j}, a_{1}^{2 j+1}, a_{0}^{j}, r_{1}^{j}\right)$, with $1 \leq j \leq i$, such that $a_{1}^{j}$ is an ancestor of $a_{1}^{i}$. We assume that the tuples in $\operatorname{Auth}\left(a_{0}^{i}\right)$ are ordered in decreasing ancestry from left to right. The signature $\sigma(m)$ on $m$ consists of $\left(\operatorname{Auth}\left(a_{0}^{i}\right), r_{0}^{i}\right)$.

\section{Verification Phase}

The receiver puts $\sigma(m) \equiv\left(\operatorname{Auth}\left(a_{0}^{j_{r}}\right), r_{0}^{j_{r}}\right)$, where $r$ is the number of tuples in $\operatorname{Auth}\left(a_{0}^{i}\right)$ and $\left(a_{1}^{j_{l}}, a_{1}^{2 j_{l}}, a_{1}^{2 j_{l}+1}, a_{0}^{j_{l}}, r_{1}^{j_{l}}\right)$ is the $l$-th tuple in $\operatorname{Auth}\left(a_{0}^{j_{r}}\right)$. After having checked whether $a_{1}^{j_{1}} \stackrel{?}{=} a_{1}^{1}$, the receiver has to perform the following verifications, for $j=2, \ldots, r$.

1. $a_{1}^{j_{2}} \stackrel{?}{\in}\left\{a_{1}^{2 j_{l-1}}, a_{1}^{2 j_{l-1}+1}\right\}$

2. $\phi\left(x_{1}, a_{1}^{j_{1}}, a_{1}^{2 j_{2}}\left\|a_{1}^{2 j_{1}+1}\right\| a_{0}^{j_{1}}, r_{1}^{j_{1}}\right) \stackrel{?}{=} 1$.

Finally, he checks whether $\phi\left(x_{0}, a_{0}^{j_{r}}, m, r_{0}^{j_{r}}\right)=1$. If all verifications hold, the signature is accepted.

Note that, by assumption on the challenge length $t(k), 2 \cdot A_{\mathcal{P}}\left(x_{1}\right)+A_{\mathcal{P}}\left(x_{0}\right) \leq t$, so the challenges are long enough to encode the strings $a_{1}^{2 i}\left\|a_{1}^{2 i+1}\right\| a_{0}^{i}$. These strings can be padded up to $t$ bits, if necessary, using standard techniques. As we have also assumed that all occurring values have fixed length descriptions (depending only on the corresponding public string), parsing these concatenations is easy.

\section{Proof of Security}

Our notion of security for signature schemes is that of [11]. In this section we show that no polynomially bounded adversary can construct a forgery on a message that hasn't been signed by the real signer, even if he is allowed to get polynomially many signatures on messages that he has chosen in an adaptive fashion. It will be shown that the existence of such a successful forger contradicts the assumption that the protocol $\mathcal{P}$ is collision intractable over the generator $G$. To this end, we compile this successful forger into an attacker that breaks that assumption. 
In the following theorem, it is assumed that we are given a signature protocol $\mathcal{P}$ for generator $G$ and relation $R$. By Theorem 2, we may assume that for each security parameter $k$ and for each instance $(x, w)$ as output by running $G\left(1^{k}\right)$, the (non-constant) polynomial $t(k)$ satisifies $t=C_{\mathcal{P}} \geq 3 \cdot A_{\mathcal{P}}$.

Theorem 8 Any probabilistic polynomial time cracking algorithm $\mathcal{A}$ that forges a signature on a new message with probability $\epsilon(k)$, after at most polynomially many calls to a signer, can be compiled into probabilistic polynomial time procedure $\mathcal{A}^{*}$ that breaks the collision intractability of $\mathcal{P}$ over $G$ with probability of the order of $\epsilon(k)$. The running time of $\mathcal{A}^{*}$ is of the same order as the running time of $\mathcal{A}$.

Proof. Let a security parameter $k$ be given, and let $x$ be an instance of $\mathcal{P}$ generated by $G$ on input $1^{k}$.

We now describe how $\mathcal{A}^{*}$ cracks the collision intractability of $\mathcal{P}$ by using the forger $\mathcal{A}$ and the following simulation of $\Sigma_{\mathcal{P}} . \mathcal{A}^{*}$ receives $x$ as input.

$\mathcal{A}^{*}$ first runs $G$ on input $1^{k}$ in order to obtain a solved instance $\left(x^{\prime}, w^{\prime}\right)$. Then a bit $b$ is chosen at random. Put $\left(x_{b}, w_{b}\right)=\left(x^{\prime}, w^{\prime}\right)$, and $x_{1-b}=x$.

For the simulation, we distinguish between two cases.

Case $b=0$ : We create an authentication tree with $P(k)$ internal nodes, starting at the leaves. The leaves $a_{1}^{j}$ are generated as follows.

1. $c^{j} \leftarrow\{0,1\}^{t}$

2. $\left(a_{1}^{j}, c^{j}, r_{1}^{j}\right) \leftarrow \mathcal{S}\left(x_{1}, c^{j}\right)$.

For children $a_{1}^{2 i}$ and $a_{1}^{2 i+1}$, generate $a_{0}^{i} \leftarrow P_{a}\left(x_{0}, w_{0}\right)$. Then the parent $a_{1}^{i}$ will be generated as

$$
\left(a_{1}^{i}, a_{1}^{2 i}\left\|a_{1}^{2 i+1}\right\| a_{0}^{i}, r_{1}^{i}\right) \leftarrow \mathcal{S}\left(x_{1}, a_{1}^{2 i}\left\|a_{1}^{2 i+1}\right\| a_{0}^{i}\right) .
$$

The resulting instance $\left(x_{0}, x_{1}, a_{1}^{1}\right)$ of $\Sigma_{\mathcal{P}}$ is sent to the forger $\mathcal{A}$. After this, the cracking algorithm can start making its (at most $P(k)$ ) calls.

The above takes care of $\operatorname{Auth}\left(a_{0}^{i}\right)$, for $i=1, \ldots, P(k)$. Note that this simulation can now deal with any signature request, as the $i$-th signature, on a message $m^{i}$, can be completed by computing $r_{0}^{i} \leftarrow P_{r}\left(x_{0}, w_{0} ; a_{0}^{i}, \operatorname{aux}\left(a_{0}^{i}\right), m^{i}\right)$.

Case $b=1$ :

1. Generate $\left(a_{1}^{1}, \operatorname{aux}\left(a_{1}^{1}\right)\right) \leftarrow P_{a}\left(x_{1}, w_{1}\right)$, and send the instance $\left(x_{0}, x_{1}, a_{1}^{1}\right)$ to the forger $\mathcal{A}$.

2. Let $m^{i} \in\{0,1\}^{t}$ be the $i$-th message to be signed. Generate $a_{0}^{i} \leftarrow \mathcal{S}\left(x_{0}, m^{i}\right)$. Proceed as in Step 3 of the signing phase of $\Sigma_{\mathcal{P}}$.

Note that in both cases the simulation can deal with any signature request, by the properties of the special simulator $\mathcal{S}$. Furthermore, the distribution of the $a_{0}^{i}, r_{0}^{i}, a_{1}^{i}$ and $r_{1}^{i}$ is always according to the honest signer who has access to both $w_{0}$ and $w_{1}$, by Theorem 1 . Thus the simulation is perfect, and we may now assume that the cracking algorithm outputs a forgery on a new message (i.e, a message that has not been signed by the simulator) $\tilde{m}$. Without loss of 
generality, we assume that this happens after exactly $P(k)$ calls, with probability $\epsilon(k)$.

Let $\left(\operatorname{Auth}\left(a_{0}\right), r_{0}\right)$ be the forgery, on a new message $\tilde{m}$. Suppose that $a_{0}=a_{0}^{j}$ for some $1 \leq j \leq P(k)$, with probability $\epsilon_{1}(k)$. As $\tilde{m}$ has not been signed by the simulation, we must have $\tilde{m} \neq m^{j}$, so $\mathcal{A}^{*}$ can get a collision for $\mathcal{P}$ from $\left(a_{0}, \tilde{m}, r_{0}\right)$ and $\left(a_{0}^{j}, m^{j}, r_{0}^{j}\right)$.

If, on the contrary, $a_{0} \neq a_{0}^{j}$ for all $1 \leq j \leq P(k)$, then there clearly exist a tuple $\left(a_{1}^{\prime}, a_{1}^{\prime \prime}, a_{1}^{\prime \prime \prime}, a_{0}^{\prime}, r_{1}^{\prime}\right)$ in Auth $\left(a_{0}\right)$ and a node $a_{1}^{i}$ in the tree, with $a_{1}^{\prime}=a_{1}^{i}$, such that $a_{1}^{i}$ is a leaf or $a_{1}^{i}$ is an internal node with $a_{1}^{\prime \prime}\left\|a_{1}^{\prime \prime \prime}|| a_{0}^{\prime} \neq a_{1}^{2 i}\right\| a_{1}^{2 i+1} \| a_{0}^{i}$.

In case $a_{1}^{i}$ is an internal node, say with probability $\epsilon_{2}(k)$, we immediately get a collision. If $a_{1}^{i}$ is a leaf, with probability $\epsilon_{3}(k)$, however, the probability that $a_{1}^{\prime \prime}\left\|a_{1}^{\prime \prime \prime}\right\| a_{0}^{\prime} \neq c^{i}$ is $1-\frac{1}{2^{i}}$, as the distribution of $a_{1}^{i}$ is independent of the distribution of $c^{j}$ (by the properties of the special simulator), and $c^{j}$ was chosen uniformly at random. Thus in this case we get a collision with probability $1-$ $\frac{1}{2^{1}}$. From the perfectness of the simulation it follows that the distribution of everything sent to $\mathcal{A}$ is independent of $b$. Therefore the probability that $\mathcal{A}^{*}$ can compute a collision for the instance $x_{1-b}=x$ is

$$
\frac{1}{2} \epsilon_{1}(k)+\frac{1}{2} \epsilon_{2}(k)+\frac{1}{2}\left(1-\frac{1}{2^{t}}\right) \epsilon_{3}(k) \geq \frac{1}{2} \epsilon(k)-\frac{1}{2^{t+1}} \epsilon_{3}(k),
$$

which is clearly of the same order as $\epsilon(k)$. Thus we have shown that any forger of the signature scheme $\Sigma_{\mathcal{P}}$ can be turned very efficiently into a cracker of the collision intractibility of $\mathcal{P}$, with essentially the same probability of success.

\section{Concrete Examples}

We now describe a signature scheme whose security is equivalent to the difficulty of computing discrete logarithms, by applying our main construction to a suitable transformation of the discrete log based protocol of Schnorr [17]. In its basic form, this is a protocol for proving knowledge of a discrete $\log$ in a group $\mathcal{G}$ of prime order $q$. Such a group can be realized, for example as a subgroup of $\mathbf{Z}_{p}^{*}$, where $p$ is a prime, and $q$ divides $p-1$.

This protocol is a quasi-signature protocol by standard arguments. With some modifications, it can be turned into a signature protocol: we will have as input to the protocol $d$ instances instead of one, $\left(x_{1}, w_{1}\right), \ldots,\left(x_{d}, w_{d}\right)$, where $x_{i}=g^{w_{i}}$. Then the new protocol $\mathcal{P}$ goes as follows:

1. The prover chooses $z$ at random in $[0, \ldots, q)$, and sends $a=g^{z}$ to $V$.

2. The verifier chooses $c_{1}, \ldots, c_{d}$ at random in $\left[0, \ldots, 2^{\prime}\right)$, and sends them to $P$.

3. $P$ sends $r=\left(z+c_{1} w_{1}+\cdots+c_{d} w_{d}\right) \bmod q$ to $V$, and $V$ checks that $g^{r}=$ $a \cdot x_{1}^{c_{1}} \cdots x_{d}^{c_{d}}$,

where $l=\left\lfloor\log _{2}(q)\right\rfloor$. Completeness and special honest verifier zero-knowledge are clear by the same arguments as above. Collision intractability can be shown by essentially the same proof as for Theorem 3. Finally, it is clear that by choosing 
d large enough, we can get a large enough challenge length, and therefore a signature protocol.

We can now carry out our construction of $\Sigma_{\mathcal{P}}$ (see also Section 4 ). To set up an instance of $\Sigma_{\mathcal{P}}$, the signer generates two independent instances of $\mathcal{P}$, $(x, w) \equiv\left(\left(x_{1}, w_{1}\right), \ldots,\left(x_{d}, w_{d}\right)\right)$ and $(\bar{x}, \bar{w}) \equiv\left(\left(\bar{x}_{1}, \bar{w}_{1}\right), \ldots,\left(\bar{x}_{d}, \bar{w}_{d}\right)\right)$, with $x_{i}=$ $g^{w_{i}}$ and $\bar{x}_{i}=g^{\bar{w}_{i}}$ for $i=1, \ldots, d$. The $w_{i}$ and $\bar{w}_{i}$ are chosen at random from $\boldsymbol{Z}_{q}$. Note that both these instances use the same pair $(g, \mathcal{G})$. The root of the authentication tree, $a_{1}^{1}$, is computed as $a_{1}^{1}=g^{z_{1}^{1}}$, where $z_{1}^{1}$ is chosen at random from $\boldsymbol{Z}_{q}$. The initialization phase of $\Sigma_{\mathcal{P}}$ is completed when the public key of the signer, $\left(x, \bar{x}, a_{1}^{1}\right)$, is placed in the public directory.

We will now show how the signer computes the first signature on a message $m \in\{0,1\}^{d \cdot I}$, where $m=m_{1}\|\ldots\| m_{d}$ and the $m_{i}$ are $l$-bitstrings, to be interpreted as members of $\left[0 \ldots 2^{l}\right)$.

First, he computes $a_{0}^{1}$ as $a_{0}^{1}=g^{z_{0}^{1}}$, with $z_{0}^{1}$ chosen at random from $\boldsymbol{Z}_{q}$, and $r_{0}^{1}$ as $r_{0}^{1}=z_{0}^{1}+m_{1} w_{1}+\cdots+m_{d} w_{d}$. Before establishing an authentication for $a_{0}^{1}$, he computes $a_{1}^{2}$ and $a_{1}^{3}$ (in the same way as $a_{1}^{1}$ ). Next, $a_{0}^{1}$ is authenticated, together with $a_{1}^{2}$ and $a_{1}^{3}$, by computing $r_{1}^{1}$ as $r_{1}^{1}=z_{1}^{1}+\mu_{1} \bar{w}_{1} \cdots+\mu_{d} \bar{w}_{d}$, where $\mu_{1}\|\cdots\| \mu_{d}=a_{1}^{2}\left\|a_{1}^{3}\right\| a_{0}^{1}$. The $\mu_{i}$ are l-bitstrings, to be interpreted as members of $\left[0, \ldots, 2^{l}\right)$.

The values $r_{0}^{1}, r_{1}^{1}, a_{0}^{1}, a_{1}^{2}$ and $a_{1}^{3}$ are forwarded to the receiver, who checks whether

$$
\begin{aligned}
& \text { 1. } g^{r_{0}^{1}} \stackrel{?}{=} a_{0}^{1} \cdot x_{1}^{m_{1}} \cdots x_{d}^{m_{d}}, \text { and } \\
& \text { 2. } g^{r_{1}^{1}} \stackrel{?}{=} a_{1}^{1} \cdot \bar{x}_{1}^{\mu_{1}} \cdots \bar{x}_{d}^{\mu_{d}} .
\end{aligned}
$$

Note that the values $a_{1}^{2}$ and $a_{1}^{3}$ are ready to play the role of $a_{1}^{1}$ in the second and third execution of $\Sigma_{\mathcal{P}}$, i.e., to authenticate $a_{0}^{2}, a_{1}^{4}, a_{1}^{5}$, and $a_{0}^{3}, a_{1}^{6}, a_{1}^{7}$, respectively.

The $i$-th signature $(i>1)$ consists of $a_{0}^{i}, r_{0}^{i}$ and an authentication path for $a_{0}^{i}$, which is a list of all tuples $\left(a_{1}^{j}, a_{1}^{2 j}, a_{1}^{2 j+1}, a_{0}^{j}, r_{1}^{j}\right)$ such that $a_{1}^{j}$ is an ancestor of $a_{1}^{i}$.

For example, such a list for $a_{0}^{11}$ would effectively consist of $a_{1}^{1}, a_{1}^{2}, a_{1}^{3}, a_{1}^{4}, a_{1}^{5}$, $a_{1}^{10}, a_{1}^{11}, a_{0}^{1}, a_{0}^{2}, a_{0}^{5}, r_{0}^{1}, r_{0}^{2}$ and $r_{0}^{5}$. Note that verification of the path requires only three exponentiations in $\mathcal{G}$.

We get signatures of length $O(k \log i)$ bits, where $k$ is the number of bits needed to represent an element in $\mathcal{G}$, and $i$ indicates the number of signatures made. Moreover, one authentication step requires a constant number of exponentiations in $\mathcal{G}$, both for signing and verification. Note that the 1 exponentiation needed from the signer uses input independent from the bits authenticated $\left(c_{1}, \ldots, c_{d}\right)$. Therefore we can use the idea suggested by Schnorr of having the signer precompute this exponentiation if some idle time is available on his computer. This way the on-line time to generate a signature becomes almost negligible.

Previously, the only known way to get a signature scheme provably secure based on discrete log was to use the method from [4] to build a collision intractable hash function and then use Merkle's construction. This would require 
an exponentiation for each bit processed in the hashing, and moreover we would need as a part of the signature a full preimage under the hash function to authenticate 1 bit. Therefore we would get signatures of length $O\left(k^{2} \log i\right)$ bits and would need $O\left(k^{2} \log i\right)$ exponentiations to make a signature.

\section{Conclusion}

We have shown that the existence of signature protocols is a sufficient condition for the existence of signature schemes that are not existentially forgeable under adaptively chosen message attacks, which is the strongest notion of security for signature schemes (see [11]). The length of the signatures in our schemes grows logarithmically in the number of signatures. In addition to the existence of claw-free pairs of trapdoor permutations, on which the scheme from [11] is based, the most general computational assumption we have been able to find, sufficient for the existence of signature protocols, is the existence of one-way group homomorphisms. As an example, we have presented a signature scheme whose security is equivalent to the difficulty of computing discrete logarithms.

\section{Acknowledgements}

It's a pleasure to thank Berry Schoenmakers for many valuable discussions and comments. Also thanks to Matt Franklin for commenting on an earlier version of this paper.

\section{References}

1. M. Abadi, E. Allender, A. Broder, J. Feigenbaum and L. Hemachandra: On Generating Solved Instances of Computational Problems, Proc. of Crypto 88, Springer Verlag LNCS series.

2. J.Bos and D.Chaum: Provably Unforgeable Signatures, Proc. of Crypto 92, Springer Verlag LNCS series.

3. E. F. Brickell and K. S. McCurley: An Interactive Identification Scheme Based on Discrete Logarithms and Factoring, Journal of Cryptology, 5(1), pp.29-39, 1992.

4. I. Damgård: Collision Free Hash Functions and Public-Key Signature Schemes, Proc. of EuroCrypt 87 , Springer Verlag LNCS series.

5. R. Cramer, I. Damgård: Secure Signature Schemes based on Interactive Protocols, BRICS report series, RS-94-29, September 1994, Aarhus University.

6. R. Cramer: On Shared Randomness and the Size of Secure Signatures, CWI technical report CS-R9530, April 1995.

7. C. Dwork, M. Naor: An Efficient Existentially Unforgeable Signature Scheme and its Applications, Proceedings of Crypto'94, Santa Barbara, August 1994, Springer Verlag LNCS series, pp. 234-246.

8. U.Feige, A.Shamir: Witness Indistinguishable and Witness Hiding Protocols, Proc. of STOC 90.

9. U. Feige, A. Fiat and A. Shamir: Zero-Knowledge Proofs of Identity, Journal of Cryptology 1 (1988) 77-94. 
10. O. Goldreich: Two Remarks concerning the GMR Signature Scheme, Proc. of Crypto 86, Springer Verlag LNCS series.

11. S. Goldwasser, S. Micali and R. Rivest: A Digital Signature Scheme Secure Against Chosen Message Attacks, SIAM Journal on Computing, 17(2): 281-308, 1988.

12. L. Guillou and J.-J. Quisquater: A Practical Zero-Knowledge Protocol fitted to Security Microprocessor Minimizing both Transmission and Memory, Proc. of EuroCrypt '88, Springer Verlag LNCS series.

13. R.C.Merkle: A Digital Signature Based on a Conventional Encryption Function, Proc. of Crypto 87, Springer Verlag LNCS series.

14. M.Naor and M.Yung: Universal One-Way Hash Functions and their Cryptographic Applications, Proc. of STOC 89.

15. T. Okamoto: Provably Secure and Practical Identification Schemes and Corresponding Signature Schemes, Proc. of Crypto '92, pp.31-53, Santa Barbara, August 1992.

16. J.Rompel: One-Way Functions are Necessary and Sufficient for Secure Signatures, Proc. of STOC 90.

17. C.P. Schnorr: Efficient Signature Generation by Smart Cards, Journal of Cryptology, 4(3):161-174, 1991.

18. M.Tompa and H.Woll: Random Self-Reducibility and Zero-Knowledge Proof of Information Possession, Proc. of FOCS 87. 